\title{
Stage IIIC Eyelid Carcinoma AJCC v7
}

National Cancer Institute

\section{Source}

National Cancer Institute. Stage IIIC Eyelid Carcinoma A/CC v7. NCI Thesaurus. Code C88132.

Stage IIIC includes: T4, Any N, M0. T4: Tumor is not resectable due to extensive invasion of ocular, orbital, craniofacial structures, or brain. M0: No distant metastasis. (AJCC 7th ed.) 